\title{
A Cauchy-type problem with a sequential fractional derivative in the space of continuous functions
}

\author{
Khaled M Furati
}

\section{Correspondence: kmfurati@kfupm. edu.sa \\ Department of Mathematics \& Statistics, King Fahd University of Petroleum \& Minerals, Dhahran \\ 31261, Saudi Arabia}

\begin{abstract}
A Cauchy-type nonlinear problem for a class of fractional differential equations with sequential derivatives is considered in the space of weighted continuous functions. Some properties and composition identities are derived. The equivalence with the associated integral equation is established. An existence and uniqueness result of the global continuous solution is proved.

AMS Subject Classification: 26A33; 34A08; 34A34; 34A12; 45J08.

Keywords: fractional derivatives, Riemann-Liouville fractional derivative, sequential fractional derivative, fractional differential equation
\end{abstract}

\section{Introduction}

We consider a Cauchy-type problem associated with the equation

$$
D_{a}^{\alpha}\left[(x-a)^{r} D_{a}^{\beta} y(x)\right]=f(x, y), \quad x>a, \quad 0<\alpha<1, \quad 0 \leq \beta \leq 1, \quad r<\alpha,
$$

where $D_{a}^{\alpha}$ and $D_{a}^{\beta}$ are the Riemann-Liouville fractional derivatives.

In recent years there has been a considerable interest in the theory and applications of fractional differential equations. As for the theory, the investigations include the existence and uniqueness of solutions, asymptotic behavior, stability, etc. See for example the books [1-3] and the articles [4-10] and the references therein.

As for the applications, fractional models provide a tool for capturing and understanding complex phenomena in many fields. See for example the surveys in $[1,11]$ and the collection of applications in [12].

Some recent applications include control systems [13,14], viscoelasticity [15-18], and nanotechnology [19]. Also fractional models are used to model a vibrating string [20], and anomalous transport [21], anomalous diffusion [22-24].

Another field of applications is in random walk and stochastic processes [25-27] and its applications in financial modeling [28-30]. Other physical and engineering processes are given in [31,32]

In a series of articles, [33-35], Glushak studied the uniform well-posedness of a Cauchy-type problem with two fractional derivatives and bounded operator. He also proposed a criterion for the uniform correctness of unbounded operator.

(c) 2012 Furati; licensee Springer. This is an Open Access article distributed under the terms of the Creative Commons Attribution License (http://creativecommons.org/licenses/by/2.0), which permits unrestricted use, distribution, and reproduction in any medium, provided the original work is properly cited. 
In this article we prove an existence and uniqueness result for a nonlinear Cauchytype problem associated with the Equation (1) in the space of weighted continuous functions.

We start with some preliminaries in Section 2. In Section 3 we define the sequential derivative and develop some properties and composition identities. In Section 4 we set up the Cauchy-type problem and establish the equivalence with the associated integral equation. Finally, in Section 5 we prove the existence and uniqueness of the solution.

\section{Preliminaries}

In this section we present some definitions, lemmas, properties and notation which we use later. For more details please see [1].

Let $-\infty<a<b<\infty$. Let $C[a, b]$ denote the spaces of continuous functions on $[a, b]$. We denote by $L(a, b)$ the spaces of Lebesgue integrable functions on $(a, b)$. Let $C L(a$, $b)=L(a, b) \cap C(a, b]$.

We introduce the weighted spaces of continuous functions

$$
C_{\gamma}[a, b]=\left\{f:(a, b] \rightarrow \mathbb{R}:(x-a)^{\gamma} f(x) \in C[a, b]\right\}, \quad \gamma \in \mathbb{R},
$$

with the norm

$$
\|f\|_{C_{\gamma}[a, b]}=\left\|(x-a)^{\gamma} f(x)\right\|_{C[a, b]}
$$

where

$$
\|f\|_{C[a, b]}=\max _{x \in[a, b]}|f(x)| .
$$

In the case $f$ is not defined at $x=a$ or $\gamma<0$ we let $\left.(x-a)^{\gamma} f(x)\right|_{x=a}=\lim _{x-a^{+}}(x-a)^{\gamma} f(x)$. The spaces $C_{\gamma}[a, b]$ satisfy the following properties.

- $C_{0}[a, b]=C[a, b]$.

- $C_{\gamma_{1}}[a, b] \subset C_{\gamma_{2}}[a, b], \gamma_{1}<\gamma_{2}$.

- $C_{\gamma}[a, b] \subset C L(a, b), \gamma<1$.

- $f \in C_{\gamma}[a, b]$ if and only if $f \in C(a, b)$ and $\lim _{x \rightarrow a^{+}}(x-a)^{\gamma} f(x)$ exists and is finite.

The left-sided Riemann-Liouville fractional integrals and derivatives are defined as follows.

Definition 1 Let $f \in L(a, b)$. The integral

$$
I_{a}^{\alpha} f(x):=\frac{1}{\Gamma(\alpha)} \int_{a}^{x} \frac{f(s)}{(x-s)^{1-\alpha}} d s, \quad x>a, \quad \alpha>0,
$$

is called the left-sided Riemann-Liouville fractional integral of order $\alpha$ of the function $f$.

Definition 2 The expression

$$
D_{a}^{\alpha} f(x):=D I_{a}^{\alpha-1} f(x), \quad x>a, \quad 0<\alpha<1, \quad D=\frac{d}{d x},
$$


is called the left-sided Riemann-Liouville fractional derivative of order $\alpha$ of $f$ provided the right-hand side exists.

For power functions we have the following formulas.

Lemma 3 For $x>a$ we have

$$
\begin{aligned}
& {\left[I_{a}^{\alpha}(t-a)^{\beta-1}\right](x)=\frac{\Gamma(\beta)}{\Gamma(\beta+\alpha)}(x-a)^{\beta+\alpha-1}, \quad \alpha \geq 0, \quad \beta>0 .} \\
& {\left[D_{a}^{\alpha}(t-a)^{\alpha-1}\right](x)=0, \quad 0<\alpha<1 .}
\end{aligned}
$$

Next we present some mapping properties of the operator $I_{a}^{\alpha}$.

Lemma 4 For $\alpha>0$, $I_{a}^{\alpha}$ maps $L(a, b)$ into $L(a, b)$.

The proof of Lemma 4 is given in [36]. The following lemma is proved in [37].

Lemma 5 For $\alpha>0, I_{a}^{\alpha}$ maps $C[a, b]$ into $C[a, b]$.

The following lemma is proved in [38].

Lemma 6 Let $\alpha \geq 0$. If $f \in C L(a, b)$ then $I_{a}^{\alpha} f \in C L(a, b)$.

The mapping properties of $I_{a}^{\alpha}$ in the spaces $C_{\gamma}[a, b], 0 \leq \alpha \leq \gamma<1$, are given in [1], Lemma 2.8 which is proved in [39] in Russian. For completeness we present here a more general result for $\alpha>0$ and $\gamma<1$. First we prove the necessity condition at the left end.

Lemma 7 Let $\alpha \geq 0$ and $\gamma<1$. If $f \in C_{\gamma}[a, b]$ then

$$
\lim _{x \rightarrow a^{+}}(x-a)^{\gamma-\alpha} I_{a}^{\alpha} f(x)=\frac{c \Gamma(1-\gamma)}{\Gamma(1+\alpha-\gamma)},
$$

where $c=\lim _{x \rightarrow a^{+}}(x-a)^{\gamma} f(x)$.

Proof. Note that from Lemma 3 we have

$$
I_{a}^{\alpha}(x-a)^{-\gamma}=\frac{\Gamma(1-\gamma)}{\Gamma(1+\alpha-\gamma)}(x-a)^{\alpha-\gamma} .
$$

Thus

$$
\begin{aligned}
\left|(x-a)^{\gamma-\alpha} I_{a}^{\alpha} f(x)-\frac{c \Gamma(1-\gamma)}{\Gamma(1+\alpha-\gamma)}\right| & =\left|(x-a)^{\gamma-\alpha} I_{a}^{\alpha} f(x)-c(x-a)^{\gamma-\alpha} I_{a}^{\alpha}(x-a)^{-\gamma}\right| \\
& =(x-a)^{\gamma-\alpha}\left|I_{a}^{\alpha} f(x)-c I_{a}^{\alpha}(x-a)^{-\gamma}\right| \\
& =(x-a)^{\gamma-\alpha}\left|I_{a}^{\alpha}\left[(x-a)^{-\gamma}\left\{(x-a)^{\gamma} f(x)-c\right\}\right]\right| .
\end{aligned}
$$

Now, given $\epsilon>0$ there exists $\delta>0$ such that $x-a<\delta$ implies that

$$
\left|(x-a)^{\gamma} f(x)-c\right|<\varepsilon \frac{\Gamma(1+\alpha-\gamma)}{\Gamma(1-\gamma)} .
$$

Thus

$$
\begin{aligned}
\left|(x-a)^{\gamma-\alpha} I_{a}^{\alpha} f(x)-\frac{c \Gamma(1-\gamma)}{\Gamma(1+\alpha-\gamma)}\right| & =(x-a)^{\gamma-\alpha}\left|I_{a}^{\alpha}\left[(x-a)^{-\gamma}\left\{(x-a)^{\gamma} f(x)-c\right\}\right]\right| \\
& <\varepsilon \frac{\Gamma(1+\alpha-\gamma)}{\Gamma(1-\gamma)}(x-a)^{\gamma-\alpha} I_{a}^{\alpha}\left[(x-a)^{-\gamma}\right] \\
& =\varepsilon \frac{\Gamma(1+\alpha-\gamma)}{\Gamma(1-\gamma)}(x-a)^{\gamma-\alpha} \frac{\Gamma(1-\gamma)}{\Gamma(1+\alpha-\gamma)}(x-a)^{\alpha-\gamma} \\
& =\varepsilon .
\end{aligned}
$$


This yields the limit (9).

Next we present the mapping properties of $I_{a}^{\alpha}$ in the spaces $C_{\gamma}[a, b]$.

Lemma 8 Let $\alpha>0$ and $\gamma<1$. If $f \in C_{\gamma}[a, b]$ then $I_{a}^{\alpha} f \in C_{\gamma-\alpha}[a, b]$ and for $x \in(a$, b] we have

$$
\left|I_{a}^{\alpha} f(x)\right| \leq \frac{\Gamma(1-\gamma)}{\Gamma(1+\alpha-\gamma)}(x-a)^{\alpha-\gamma}\|f\|_{C_{\gamma}[a, b]} .
$$

Proof. From Lemmas 6 and 7 we have $I^{\alpha} f \in C(a, b)$ and $\lim _{x \rightarrow a^{+}}(x-a)^{\gamma-\alpha} I_{a}^{\alpha} f(x)$ exists and is finite. Thus $I_{a}^{\alpha} f \in C_{\gamma-\alpha}[a, b]$. Now for $x \in(a, b]$ we have

$$
\begin{aligned}
\left|I_{a}^{\alpha} f(x)\right| & =\frac{1}{\Gamma(\alpha)}\left|\int_{a}^{x}(x-t)^{\alpha-1}(t-a)^{-\gamma}(t-a)^{\gamma} f(t) d t\right| \\
& \leq \frac{1}{\Gamma(\alpha)}\|f\|_{C_{\gamma}[a, b]} \int_{a}^{x}(x-t)^{\alpha-1}(t-a)^{-\gamma} d t .
\end{aligned}
$$

The relation (10) follows by applying Lemma 3.

Consequently, from Lemma 8 we have the following property.

Lemma 9 Let $\alpha>0, \gamma<1$, and $r \in \mathbb{R}$. If $f \in C_{\gamma}[a, b]$ then $(x-a)^{-r} I_{a}^{\alpha} f \in C_{\gamma+r-\alpha}[a, b]$. In particular, if $\gamma+r-\alpha<1$ then $I^{\alpha} f \in C L(a, b)$.

Later, the following observation is important.

Lemma 10 Let $\alpha>0$ and $r<\alpha$. If $f \in C L(a, b)$ then $(x-a)^{-r} I_{a}^{\alpha} f \in C L(a, b)$.

Proof. When $r \leq 0$ the result follows clearly from Lemma 6. When $r>0$ it follows from Lemma 6 that $(x-a)^{-r} I_{a}^{\alpha} f \in C(a, b)$ and we only need to show that $(x-a)^{-r} I_{a}^{\alpha} f \in L(a, b)$.

For any $x \in(a, b]$ we have the following inequality.

$$
\begin{aligned}
\left|I_{a}^{\alpha} f(x)\right| & =\frac{1}{\Gamma(\alpha)}\left|\int_{a}^{x}(x-t)^{\alpha-1} f(t) d t\right| \\
& =\frac{1}{\Gamma(\alpha)} \int_{a}^{x}(x-t)^{\alpha-r-1}(x-t)^{r}|f(t)| d t \\
& \leq \frac{(x-a)^{r}}{\Gamma(\alpha)} \int_{a}^{x}(x-t)^{\alpha-r-1}|f(t)| d t \\
& =\frac{\Gamma(\alpha-r)}{\Gamma(\alpha)}(x-a)^{r} I_{a}^{\alpha-r}|f(x)| .
\end{aligned}
$$

Or,

$$
(x-a)^{-r}\left|I_{a}^{\alpha} f(x)\right| \leq \frac{\Gamma(\alpha-r)}{\Gamma(\alpha)} I_{a}^{\alpha-r}|f(x)| .
$$

From Lemma 4 the right-hand side is in $L(a, b)$ and thus $(x-a)^{-r} I_{a}^{\alpha} f \in L(a, b)$. This completes the proof.

The following lemma follows by direct calculations using Dirichlet formula, [36]. 
Lemma 11 Let $\alpha \geq 0, \beta \geq 0$, and $f \in C L(a, b)$. Then

$$
I_{a}^{\alpha} I_{a}^{\beta} f(x)=I_{a}^{\alpha+\beta} f(x),
$$

for all $x \in(a, b]$.

Lemma 11 leads to the left inverse operator.

Lemma 12 Let $\alpha>0$ and $f \in C L(a, b)$. Then

$$
D_{a}^{\alpha} I_{a}^{\alpha} f(x)=f(x),
$$

for all $x \in(a, b]$.

Now we present a version of the fundamental theorem of fractional calculus.

Lemma 13 Let $0<\alpha<1$. If $f \in C(a, b)$ and $D_{a}^{\alpha} f \in C L(a, b)$, then $f \in C L(a, b), I_{a}^{\alpha} f\left(a^{+}\right)$exists and is finite, and

$$
I_{a}^{\alpha} D_{a}^{\alpha} f(x)=f(x)-\frac{I_{a}^{1-\alpha} f\left(a^{+}\right)}{\Gamma(\alpha)}(x-a)^{\alpha-1},
$$

for all $x \in(a, b]$.

Proof. From Lemma 12 we have for all $x \in(a, b]$ the relation

$$
D_{a}^{\alpha} I_{a}^{\alpha} D_{a}^{\alpha} f(x)=D_{a}^{\alpha} f(x),
$$

which we can write as

$$
D_{a}^{\alpha}\left[f-I_{a}^{\alpha} D_{a}^{\alpha} f\right](x)=0 .
$$

This implies that

$$
f(x)-I_{a}^{\alpha} D_{a}^{\alpha} f(x)=c(x-a)^{\alpha-1},
$$

for some constant $c$. Since Lemma 6 implies that $I_{a}^{\alpha} D_{a}^{\alpha} f \in C L(a, b)$, we also have $f \in$ $C L(a, b)$. Also, if we apply $I_{a}^{1-\alpha}$ to both sides of (14) we obtain

$$
I_{a}^{1-\alpha} f(x)=I_{a} D_{a}^{\alpha} f(x)=c \Gamma(\alpha) .
$$

Taking the limit yields $I_{a}^{1-\alpha} f\left(a^{+}\right)=c \Gamma(\alpha)$ and (13) is obtained.

In the proof of our existence and uniqueness result we will use the following results.

Lemma 14 Let $\gamma \in \mathbb{R}, a<c<b, g \in C_{\gamma}[a, c], g \in C[c, b]$ and $g$ is continuous at $c$. Then $g \in C_{\gamma}[a, b]$.

Theorem 15 ([1], Banach Fixed Point Theorem) Let $(U, d)$ be a nonempty complete metric space. Let $T: U \rightarrow U$ be a map such that for every $u, v \in U$, the relation

$$
d(T u, T v) \leq w d(u, v), \quad 0 \leq w<1,
$$

holds. Then the operator $T$ has a unique fixed point $u^{*} \in U$.

\section{Sequential derivative}

In this section we define the sequential derivative and integral that we consider and develop some of their properties. In particular, we derive the composition identities.

Definition 16 Let $\alpha>0, \beta>0, r \in \mathbb{R}$. Let $f \in C L(a, b)$. Define the sequential integral $\mathcal{J}_{r, a}^{\alpha, \beta} f$ and the sequential derivative $\mathcal{D}_{r, a}^{\alpha, \beta} f$ by 


$$
\mathcal{J}_{r, a}^{\alpha, \beta} f(x)=I_{a}^{\alpha}(x-a)^{-r} I_{a}^{\beta} f(x),
$$

and

$$
\mathcal{D}_{r, a}^{\alpha, \beta} f(x)=D_{a}^{\alpha}(x-a)^{r} D_{a}^{\beta} f(x),
$$

if the right-hand sides exist.

From Lemma 3 we have the following formula for the power function.

Lemma 17 Let $\alpha>0, \beta>0, r \in \mathbb{R}$. If

$$
\rho>\max \{-1, \beta-r-1\},
$$

then for $x>a$,

$$
\mathcal{D}_{r, a}^{\alpha, \beta}(x-a)^{\rho}=\frac{\Gamma(\rho+1)}{\Gamma(\rho-\beta+1)} \frac{\Gamma(\rho+r-\beta+1)}{\Gamma(\rho+r-\beta-\alpha+1)}(x-a)^{\rho+r-\beta-\alpha} .
$$

Moreover, from Lemmas 3 and 17 we have the following vanishing derivatives.

\section{Lemma 18}

(a) Let $\alpha>0,0<\beta<1, r \in \mathbb{R}$. Then for $x>a$,

$$
\mathcal{D}_{r, a}^{\alpha, \beta}(x-a)^{\beta-1}=0 .
$$

(b) Let $0<\alpha<1$ and $\beta>0$. Let $r \in \mathbb{R}$ be such that $r<\alpha+\beta$. Then for $x>a$,

$$
\mathcal{D}_{r, a}^{\alpha, \beta}(x-a)^{\alpha+\beta-r-1}=0 .
$$

Lemma 19 (Left inverse) Let $\alpha>0, \beta>0$, and $r \in \mathbb{R}$. If $f \in C L(a, b)$ such that $(x-a)^{-r} I_{a}^{\alpha} f \in C L(a, b)$ then

$$
\mathcal{D}_{r, a}^{\alpha, \beta} \mathcal{J}_{r, a}^{\beta, \alpha} f(x)=f(x),
$$

for all $x \in(a, b]$.

Proof. Relation (20) follows directly by applying Lemma 12 twice.

From Lemmas 8 and 9 we have the following mapping property of the operator $\mathcal{J}_{r, a}^{\beta, \alpha}$.

Lemma 20 Let $\alpha>0, \beta>0$, and $r<1+\alpha$. Let $0 \leq \gamma<\min \{1,1+\alpha-r\}$. If $f \in C_{\gamma}[a$, b] then $\mathcal{J}_{r, a}^{\beta, \alpha} f \in C_{\gamma+r-\alpha-\beta}[a, b]$ and for $x \in(a, b]$ we have

$$
\left|\mathcal{J}_{r, a}^{\beta, \alpha} f(x)\right| \leq k\|f\|_{C_{\gamma}[a, b]}(x-a)^{\alpha+\beta-r-\gamma},
$$

where

$$
k=\frac{\Gamma(1-\gamma) \Gamma(1+\alpha-r-\gamma)}{\Gamma(1-\gamma+\alpha) \Gamma(1+\alpha+\beta-r-\gamma)} .
$$

Lemma 20 implies the following.

Lemma 21 Let $\alpha>0, \beta>0$, and $r<1+\alpha$. Let $0 \leq \gamma<\min \{1,1+\alpha-r\}$. If $r \leq \alpha+\beta$, then $\mathcal{J}_{r, a}^{\beta, \alpha}$ is bounded in $C_{\gamma}[a, b]$ and 


$$
\left\|\mathcal{J}_{r, a}^{\beta, \alpha} f\right\|_{C_{\gamma}[a, b]} \leq k(b-a)^{\alpha+\beta-r}\|f\|_{C_{\gamma}[a, b]^{\prime}}
$$

where $k$ is given by (22).

Proof. Since $\gamma+r-\alpha-\beta \leq \gamma$, then from Lemma 20 we have

$$
\mathcal{J}_{r, a}^{\beta, \alpha} f \in C_{\gamma+r-\alpha-\beta}[a, b] \subset C_{\gamma}[a, b] .
$$

The bound in (23) follows by multiply (21) by $(x-a)^{\gamma}$ and taking the maximum. As a special case of Lemma 21, we have

Lemma 22 Let $\alpha>0, \beta>0$, and $r<\min \{\alpha+1, \alpha+\beta\}$. Then $\mathcal{J}_{r, a}^{\beta, \alpha}$, maps $C[a, b]$ into $C[a, b]$ and

$$
\left\|\mathcal{J}_{r, a}^{\beta, \alpha} f\right\|_{C[a, b]} \leq L(b-a)^{\alpha+\beta-r}\|f\|_{C[a, b]^{\prime}}
$$

where

$$
L=\frac{\Gamma(1+\alpha-r)}{\Gamma(1+\alpha) \Gamma(1+\alpha+\beta-r)} .
$$

The following is an analogous result to the result for the Riemann-Liouville integral proved in [10].

Lemma 23 Let $\alpha>0, \beta>0$, and $r<\alpha$. Let $f \in C L(a, c)$. Let

$$
g(x)=\frac{1}{\Gamma(\beta)} \int_{a}^{c}(x-t)^{\beta-1}(t-a)^{-r} I_{a}^{\alpha} f(t) d t .
$$

Then

$$
\lim _{x \rightarrow c^{+}} g(x)=\mathcal{J}_{r, a}^{\beta, \alpha} f(c) .
$$

Proof. Since $r<\alpha$, Lemma 10 implies that $(x-a)^{-r} I_{a}^{\alpha} f(x) \in C L(a, c)$. Thus $\mathcal{J}_{r, a}^{\beta, \alpha} f(c)$ is finite and

$$
\left|g(x)-\mathcal{J}_{r, a}^{\beta, c} f(c)\right| \leq \frac{1}{\Gamma(\beta)} \int_{a}^{c} k(x, t)(t-a)^{-r} I_{a}^{\alpha}|f(t)| d t,
$$

where

$$
k(x, t)=\left|(c-t)^{\beta-1}-(x-t)^{\beta-1}\right| .
$$

Since $\lim _{x \rightarrow c^{+}} k(x, t)=0$, the limit of the right-hand side vanishes and the proof is complete.

The following lemma relates the fractional derivative $\mathcal{D}_{r, a}^{\alpha, \beta}$ to the Riemann-Liouville derivative $D_{a}^{\beta}$.

Lemma 24 Let $0<\alpha<1, \beta \geq 0$, and $r \in \mathbb{R}$. If $D_{a}^{\beta} \gamma(x) \in C(a, b)$ and $D_{r, a}^{\alpha, \beta} y \in C L(a, b)$ then $(x-a)^{r} D_{a}^{\beta} y \in C L(a, b), I_{a}^{1-\alpha}\left[(x-a)^{r} D_{a}^{\beta} \gamma\right]\left(a^{+}\right)$exists and finite, and 


$$
D_{a}^{\beta} \gamma(x)=(x-a)^{-r} I_{a}^{\alpha} \mathcal{D}_{r, a}^{\alpha, \beta} \gamma(x)+\frac{I^{1-\alpha}\left[(x-a)^{r} D_{a}^{\beta} \gamma\right]\left(a^{+}\right)}{\Gamma(\alpha)}(x-a)^{\alpha-r-1},
$$

for all $x \in(a, b]$. If in addition, $r<\alpha$ then $D_{a}^{\beta} y \in C L(a, b)$.

Proof. Clearly $D_{a}^{\beta} y \in C(a, b)$ implies that $(x-a)^{r} D_{a}^{\beta} y \in C(a, b)$. Thus we can apply Lemma 13 to $(x-a)^{r} D_{a}^{\beta} y$ and obtain

$$
\begin{aligned}
I_{a}^{\alpha} \mathcal{D}_{r, a}^{\alpha, \beta} \gamma(x) & =I_{a}^{\alpha} D_{a}^{\alpha}(x-a)^{r} D_{a}^{\beta} \gamma(x) \\
& =(x-a)^{r} D_{a}^{\beta} \gamma(x)-\frac{I_{a}^{1-\alpha}\left[(x-a)^{r} D_{a}^{\beta} \gamma\right]\left(a^{+}\right)}{\Gamma(\alpha)}(x-a)^{\alpha-1} .
\end{aligned}
$$

By multiplying both sides by $(x-a)^{r}$ we obtain (26). If $r<\alpha$ then Lemma 10 implies that $(x-a)^{-r} I_{a}^{\alpha} \mathcal{D}_{r, a}^{\alpha, \beta} \gamma(x) \in C L(a, b)$ and thus from (26) we have $D_{a}^{\beta} y \in C L(a, b)$. This proves the result.

The Next lemma gives an analogous result to the fundamental theorem of calculus in terms of the operators $\mathcal{D}_{r, a}^{\alpha, \beta}$ and $\mathcal{J}_{r, a}^{\alpha, \beta}$.

Lemma 25 Let $0<\alpha<1$ and $0<\beta<1$. Let $y \in C(a, b)$ be such that $\mathcal{D}_{r, a}^{\alpha, \beta} y \in C L(a, b)$ and $D_{a}^{\beta} y \in C L(a, b)$. Then both $I_{a}^{1-\alpha}\left[(x-a)^{r} D_{a}^{\beta} y\right]\left(a^{+}\right)$and $I_{a}^{1-\beta} y\left(a^{+}\right)$exist, $y \in C L(a$, b), and

$$
\begin{aligned}
\mathcal{J}_{r, a}^{\beta, \alpha} \mathcal{D}_{r, a}^{\alpha, \beta} \gamma(x)= & \gamma(x)-\frac{\left[I_{a}^{1-\beta} \gamma\right]\left(a^{+}\right)}{\Gamma(\beta)}(x-a)^{\beta-1} \\
& -\frac{\left[I_{a}^{1-\alpha}(x-a)^{r} D_{a}^{\beta} \gamma\right]\left(a^{+}\right)}{\Gamma(\alpha)} \frac{\Gamma(\alpha+r)}{\Gamma(\alpha+\beta-r)}(x-a)^{\alpha+\beta-r-1},
\end{aligned}
$$

for all $x \in(a, b]$.

Proof. By applying Lemma 13 twice we obtain

$$
\begin{aligned}
\mathcal{J}_{r, a}^{\beta, \alpha} \mathcal{D}_{r, a}^{\alpha, \beta} \gamma(x)= & I_{a}^{\beta}(x-a)^{-r} I_{a}^{\alpha} D_{a}^{\alpha}(x-a)^{r} D_{a}^{\beta} \gamma(x) \\
= & I^{\beta}(x-a)^{-r}\left[(x-a)^{r} D_{a}^{\beta} \gamma(x)-\frac{\left[I_{a}^{1-\alpha}(x-a)^{r} D_{a}^{\beta} \gamma\right]\left(a^{+}\right)}{\Gamma(\alpha)}(x-a)^{\alpha-1}\right] \\
= & I_{a}^{\beta} D_{a}^{\beta} \gamma-\frac{\left[I_{a}^{1-\alpha}(x-a)^{r} D_{a}^{\beta} \gamma\right]\left(a^{+}\right)}{\Gamma(\alpha)} I_{a}^{\beta}(x-a)^{\alpha-1-r} \\
= & y(x)-\frac{\left[I_{a}^{1-\beta} \gamma\right]\left(a^{+}\right)}{\Gamma \beta}(x-a)^{\beta-1} \\
& \quad-\frac{\left[I_{a}^{1-\alpha}(x-a)^{r} D_{a}^{\beta} \gamma\right]\left(a^{+}\right)}{\Gamma(\alpha)} \frac{\Gamma(\alpha+r)}{\Gamma(\alpha+\beta-r)}(x-a)^{\alpha+\beta-r-1} .
\end{aligned}
$$

\section{Cauchy-type problem and equivalency}

Consider the Cauchy-type problem

$$
\mathcal{D}_{r, a}^{\alpha, \beta} \gamma(x)=f(x, y(x)), \quad a<x \leq b, \quad 0<\alpha<1, \quad 0<\beta<1, r \in \mathbb{R},
$$




$$
\begin{aligned}
& \lim _{x \rightarrow a^{+}} I_{a}^{1-\alpha}\left[(x-a)^{r} D_{a}^{\beta} y\right](x)=c_{1}, \\
& \lim _{x \rightarrow a^{+}} I_{a}^{1-\beta} y(x)=c_{0},
\end{aligned}
$$

where $c_{0}$ and $c_{1}$ are real numbers.

In this problem there are two conditions even when $0<\alpha+\beta<1$. The two initial conditions are based on the composition (27). The condition (29) is of one order less than that in the differential Equation (28) while the condition (30) is one order less than the equation for $D_{a}^{\beta} \gamma$.

In addition, from [1, Lemma 3.2], the condition (30) follows from the condition

$$
\lim _{x \rightarrow a^{+}}\left[(x-a)^{1-\beta} y(x)\right]=\frac{c_{0}}{\Gamma(\beta)}
$$

and if $0<\alpha-r<1$ then (29) follows from the condition

$$
\lim _{x \rightarrow a^{+}}\left[(x-a)^{1-\alpha+r} D_{a}^{\beta} \gamma(x)\right]=\frac{c_{1}}{\Gamma(\alpha)} .
$$

Consequently, the results below hold under conditions of the type (31) and (32).

Now, Based on the composition in Lemma 24, in the next theorem we establish an equivalence with the following fractional integro-differential equation:

$$
\begin{aligned}
& D_{a}^{\beta} y=(x-a)^{-r} I_{a}^{\alpha} f[x, y(x)]+\frac{c_{1}}{\Gamma(\alpha)}(x-a)^{\alpha-r-1}, \\
& \lim _{x \rightarrow a^{+}} I_{a}^{1-\beta} y(x)=c_{0} .
\end{aligned}
$$

Theorem 26 Let $0<\alpha<1, \beta>0$ and $r \in \mathbb{R}$. Let $f:(a, b] \times \mathbb{R} \rightarrow \mathbb{R}$ be a function such that $f(., y().) \in C_{1-\alpha}[a, b]$ for any $y \in C_{1-\alpha}[a, b]$. Then we have the following.

(a) If $y \in C_{1-\alpha}[a, b]$ satisfies (33) and (34) then $y(x)$ satisfies (28-30).

(b) If $y \in C_{1-\alpha}[a, b]$ with $D_{a}^{\beta} y \in C(a, b)$ satisfy (28-30), then $y(x)$ satisfies (33-34).

\section{Proof.}

For assertion (a), let $y \in C_{1-\alpha}[a, b]$ satisfy (33-34). We multiply (33) by $(x-a)^{r}$ to obtain

$$
(x-a)^{r} D_{a}^{\beta} y=I_{a}^{\alpha} f(x, y(x))+\frac{c_{1}}{\Gamma(\alpha)}(x-a)^{\alpha-1} .
$$

Next we apply $D_{a}^{\alpha}$ to both sides of (35) to obtain (28). As for the initial condition, apply $I_{a}^{1-\alpha}$ to both sides of (35) and then take the limit to obtain (29).

For assertion (b), let $y \in C_{1-\alpha}[a, b]$ satisfy (28-30). Since $f(x, y(x)) \in C_{1-\alpha}[a, b]$, then from (28) we have $\mathcal{D}_{r, a}^{\alpha, \beta} y \in C_{1-\alpha}[a, b]$. Since also by hypothesis $D_{a}^{\beta} y \in C(a, b)$, we can apply Lemma 24 and the formula (26) holds. By substituting the initial condition we obtain (33). This completes the proof. 
The composition in Lemma 25 leads to the nonlinear integral equation,

$$
y(x)=\mathcal{J}_{r, a}^{\beta, \alpha} f(x, y(x))+\frac{c_{0}}{\Gamma(\beta)}(x-a)^{\beta-1}+\frac{c_{1} \Gamma(\alpha-r)}{\Gamma(\alpha) \Gamma(\alpha+\beta-r)}(x-a)^{\alpha+\beta-r-1} .
$$

The following theorem establishes an equivalence with this equation.

Theorem 27 Let $0<\alpha<1,0<\beta<1$ and $r<\alpha$. Let $f:(a, b] \times \mathbb{R} \rightarrow \mathbb{R}$ be a function such that $f(., y().) \in C_{1-\beta}[a, b]$ for any $y \in C_{1-\beta}[a, b]$. Then the following statements hold.

(a) If $y \in C_{1-\beta}[a, b]$ satisfies the integral Equation (36) then $y(x)$ satisfies the Cauchytype problem (28-30).

(b) If $y \in C_{1-\beta}[a, b]$ with $D_{a}^{\beta} y \in C(a, b)$ satisfies the Cauchy-type problem (28-30), then $y(x)$ satisfies the integral Equation (36).

Proof. (a). Let $y \in C_{1-\beta}[a, b]$ satisfy the integral Equation (36). By hypothesis we have $f \in C_{1-\beta}[a, b]$. Moreover, from Lemma 9 and the hypothesis $r<\alpha$, we have

$$
(x-a)^{-r} I_{a}^{\alpha} f \in C_{1+r-\alpha-\beta}[a, b] \subset C L(a, b) .
$$

Thus the hypothesis of Lemmas 18 and 19 are satisfied. Applying the operator $\mathcal{D}_{r, a}^{\alpha, \beta}$ to both sides of (36) and using Lemmas 18 and 19 yields (28) as follows.

$$
\begin{aligned}
\mathcal{D}_{r, a}^{\alpha, \beta} \gamma(x)= & \mathcal{D}_{r, a}^{\alpha, \beta} \mathcal{J}_{r, a}^{\beta, \alpha} f(x, y(x))+ \\
& \mathcal{D}_{r, a}^{\alpha, \beta}\left[\frac{c_{0}}{\Gamma(\beta)}(x-a)^{\beta-1}+\frac{c_{1} \Gamma(\alpha-r)}{\Gamma(\alpha) \Gamma(\alpha+\beta-r)}(x-a)^{\alpha+\beta-r-1}\right] \\
= & f(x, y(x)) .
\end{aligned}
$$

Next, applying $I_{a}^{1-\beta}$ to both sides of (36) yields

$$
I_{a}^{1-\beta} \gamma(x)=\mathcal{J}_{r, a}^{1, \alpha} f(x, y(x))+c_{0}+\frac{c_{1}}{\Gamma(\alpha)} \frac{\Gamma(\alpha-r)}{\Gamma(\alpha+\beta-r)} \frac{\Gamma(\alpha+\beta-r)}{\Gamma(\alpha-r+1)}(x-a)^{\alpha-r}
$$

Since $r<\alpha$, taking the limit we obtain the initial condition (30).

Applying $I_{a}^{1-\alpha}(x-a)^{r} D_{a}^{\beta}$ to both sides of (36) and using Lemmas 3, 11, and 12 yields

$$
\begin{aligned}
I_{a}^{1-\alpha}(x-a)^{r} D_{a}^{\beta} y(x) & =I_{a}^{1-\alpha}(x-a)^{r} D_{a}^{\beta}\left[\mathcal{J}_{r, a}^{\beta, \alpha} f(x, y(x))\right]+ \\
& I_{a}^{1-\alpha}(x-a)^{r} D_{a}^{\beta}\left[\frac{c_{1} \Gamma(\alpha-r)}{\Gamma(\alpha) \Gamma(\alpha+\beta-r)}(x-a)^{\alpha+\beta-r-1}\right] \\
& =I_{a}^{1} f(x, \gamma(x))+c_{1} .
\end{aligned}
$$

Again, taking the limit we obtain the initial condition (29).

(b). Let $y \in C_{1-\beta}[a, b]$ satisfy (28-30). Since $f(x, y(x)) \in C L(a, b)$ then from (28), $\mathcal{D}_{r, a}^{\alpha, \beta} y \in C L(a, b)$. Since $r<\alpha$ then from Lemma 24 we have $D_{a}^{\beta} y \in C L(a, b)$. Thus we can apply Lemma 25 and the formula (27) holds. By using the initial conditions we obtain (36). This completes the proof.

In the next section we use this equivalence to prove the existence and uniqueness of solutions. 


\section{Existence and uniqueness of the solution of the Cauchy-type problem}

In this section we prove an existence and uniqueness result for the Cauchy-type problem (28-30) using the integral Equation (36). For this purpose we introduce the following lemma.

Lemma 28 Let $0<r<\alpha<1,0<\beta<1$, then the fractional differentiation operator $\mathcal{J}_{r, a}^{\beta, \alpha}$ is bounded in $C_{1-\beta}[a, b]$ and

$$
\left\|\mathcal{J}_{r, a}^{\beta, \alpha} f\right\|_{C_{1-\beta}[a, b]} \leq K(b-a)^{\alpha+\beta-r}\|f\|_{C_{1-\beta}[a, b]^{\prime}}
$$

where

$$
K=\frac{\Gamma(\beta) \Gamma(\alpha+\beta-r)}{\Gamma(\alpha+\beta) \Gamma(\alpha+2 \beta-r)} .
$$

Proof. Clearly from the hypothesis we have $r<\alpha+\beta$ and $0<1-\beta<\min \{1,1+\alpha-$ $r$ \}. Thus the result follows by taking $\gamma=1-\beta$ in Lemma 21 .

Theorem 29 Let $0<r<\alpha<1,0 \leq \beta<1$. Let $f:(a, b] \times \mathbb{R} \rightarrow \mathbb{R}$ be a function such that $f(., y().) \in C_{1-\beta}[a, b]$ for any $y \in C_{1-\beta}[a, b]$ and the condition:

$$
\left|f\left(x, y_{1}\right)-f\left(x, y_{2}\right)\right| \leq A\left|y_{1}-y_{2}\right|, \quad A>0,
$$

is satisfied for all $x \in(a, b]$ and for all $y_{1}, y_{2} \in \mathbb{R}$.

Then the Cauchy-type problem (28-30) has a solution $y \in C_{1-\beta}[a, b]$. Furthermore, if for this solution $D_{a}^{\beta} y \in C(a, b)$, then this solution is unique.

\section{Proof.}

According to Theorem 27(a), we can consider the existence of an $C_{1-\beta}[a, b]$ solution for the integral Equation (36). This equation holds in any interval $\left(a, x_{1}\right] \subset(a, b], a$ $<x_{1}<b$. Choose $x_{1}$ such that

$$
w_{1}:=A K\left(x_{1}-a\right)^{\alpha+\beta-r}<1,
$$

where $K$ is given by (39). We rewrite the integral equation in the form $y(x)=T y(x)$, where

$$
\operatorname{Ty}(x)=v_{0}(x)+\mathcal{J}_{r, a}^{\beta, \alpha} f(x, y(x)),
$$

and

$$
v_{0}(x)=\frac{c_{0}}{\Gamma(\beta)}(x-a)^{\beta-1}+\frac{c_{1} \Gamma(\alpha-r)}{\Gamma(\alpha) \Gamma(\alpha+\beta-r)}(x-a)^{\alpha+\beta-r-1} .
$$

Since $r<\alpha$ then $v_{0} \in C_{1-\beta}[a, b]$. Thus, it follows from Lemma 28 that if $y \in C_{1-\beta}[a$, $\left.x_{1}\right]$ then $T y \in C_{1-\beta}\left[a, x_{1}\right]$. Also, for any $y_{1}, y_{2}$ in $C_{1-\beta}\left[a, x_{1}\right]$, we have

$$
\begin{aligned}
\left\|T y_{1}-T y_{2}\right\|_{C_{1-\beta}\left[a, x_{1}\right]} & \leq\left\|\mathcal{J}_{r, a}^{\beta, \alpha}\left\{\left|f\left(x, y_{1}(x)\right)-f\left(x, y_{2}(x)\right)\right|\right\}\right\|_{C_{1-\beta}\left[a, x_{1}\right]} \\
& \leq A\left\|\mathcal{J}_{r, a}^{\beta, \alpha}\left\{\left|y_{1}(x)-y_{2}(x)\right|\right\}\right\|_{C_{1-\beta}\left[a, x_{1}\right]} \\
& \leq A K\left(x_{1}-a\right)^{\alpha+\beta-r}\left\|y_{1}-y_{2}\right\|_{C_{1-\beta}\left[a, x_{1}\right]} \\
& \leq w_{1}\left\|y_{1}-y_{2}\right\|_{C_{1-\beta}\left[a, x_{1}\right]^{\prime}} \quad 0<w_{1}<1 .
\end{aligned}
$$


Hence by Theorem 15 there exists a unique solution $y^{*} \in C_{1-\beta}\left[a, x_{1}\right]$ to the Equation (36) on the interval $\left(a, x_{1}\right]$.

If $x_{1} \neq b$ then we consider the interval $\left[x_{1}, b\right]$. On this interval we consider solutions $y \in C\left[x_{1}, b\right]$ for the equation

$$
y(x)=T y(x):=v_{01}(x)+\mathcal{J}_{r, x_{1}}^{\beta, \alpha} f(x, y(x)), \quad x \in\left[x_{1}, b\right],
$$

where

$$
\begin{aligned}
v_{01}(x)= & \frac{y_{0}}{\Gamma(\beta)}(x-a)^{\beta-1}+ \\
& \frac{1}{\Gamma(\alpha) \Gamma(\beta)} \int_{a}^{x_{1}}\left[(x-t)^{\beta-1}(t-a)^{-r}\left\{\int_{a}^{t}(t-s)^{\alpha-1} f(s, \gamma(s)) d s\right\}\right] d t .
\end{aligned}
$$

Now we select $x_{2} \in\left(x_{1}, b\right]$ such that

$$
w_{2}:=A L\left(x_{2}-x_{1}\right)^{\alpha+\beta-r}<1,
$$

where $L$ is given by (25). Since the solution is uniquely defined on the interval ( $a$, $\left.x_{1}\right]$, we can consider $v_{01}(x)$ to be a known function. For $y_{1}, y_{2} \in C\left[x_{1}, x_{2}\right]$, it follows from the Lipschitz condition and Lemma 22 that

$$
\begin{aligned}
\left\|T y_{1}-T y_{2}\right\|_{C\left[x_{1}, x_{2}\right]} & \leq\left\|\mathcal{J}_{r, x_{1}}^{\beta, \alpha}\left\{\left|f\left(x, y_{1}(x)\right)-f\left(x, y_{2}(x)\right)\right|\right\}\right\|_{C\left[x_{1}, x_{2}\right]} \\
& \leq A\left\|\mathcal{J}_{r, x_{1}}^{\beta, \alpha}\left\{\left|y_{1}(x)-y_{2}(x)\right|\right\}\right\|_{C\left[x_{1}, x_{2}\right]} \\
& \leq A L\left(x_{2}-x_{1}\right)^{\alpha+\beta-r}\left\|y_{1}-y_{2}\right\|_{C\left[x_{1}, x_{2}\right]} \\
& \leq w_{2}\left\|y_{1}-y_{2}\right\|_{C\left[x_{1}, x_{2}\right]} .
\end{aligned}
$$

Since $0<w_{2}<1, T$ is a contraction. Since $f(x, y(x)) \in C\left[x_{1}, x_{2}\right]$ for any $y \in C\left[x_{1}, x_{2}\right]$, then $\mathcal{J}_{r, x_{1}}^{\beta, \alpha} f \in C\left[x_{1}, x_{2}\right]$. Moreover, clearly $v_{01}(x)$ is in $C\left[x_{1}, x_{2}\right]$. Thus the right-hand side of (41) is in $C\left[x_{1}, x_{2}\right]$. Therefore $T$ maps $C\left[x_{1}, x_{2}\right]$ into itself. By Theorem 15, there exists a unique solution $y_{1}^{*} \in C\left[x_{1}, x_{2}\right]$ to the equation on the interval $\left[x_{1}, x_{2}\right]$. Moreover, it follows from Lemma 23 that $y_{1}^{*}\left(x_{1}\right)=y_{0}^{*}\left(x_{1}\right)$. Therefore if

$$
y^{*}(x)= \begin{cases}y_{0}^{*}(x), & a<x \leq x_{1} \\ y_{1}^{*}(x), & x_{1}<x \leq x_{2}\end{cases}
$$

then by Lemma $14, y^{*} \in C_{1-\beta}\left[a, x_{2}\right]$. So $y^{*}$ is the unique solution of (36) in $C_{1-\beta}\left[a, x_{2}\right]$ on the interval $\left(a, x_{2}\right]$.

If $x_{2} \neq b$, we repeat the process as necessary, say $M-2$ times, to obtain the unique solutions $y_{k}^{*} \in C_{1-\beta}\left[x_{k}, x_{k+1}\right], k=2,3, \ldots, M$, where $a=x_{0}<x_{1}<\cdots<x_{M}=b$, such that

$$
w_{k+1}=A L\left(x_{k+1}-x_{k}\right)^{\alpha+\beta-r}<1 \text {. }
$$

As a result we have the unique solution $y^{*} \in C_{1-\beta}[a, b]$ of (36) given by

$$
y^{*}(x)=y_{k}^{*}(x), \quad x \in\left(x_{k}, x_{k+1}\right], \quad k=0,1, \ldots, M-1 .
$$

This solution is also a solution for (28-30). 


\section{If $D_{a}^{\beta} y \in C(a, b)$ then the uniqueness follows from part (b) of Theorem 27. This completes the proof.}

\section{Acknowledgements}

The author was grateful for the support provided by the King Fahd University of Petroleum \& Minerals and the financial support by the BAE Systems through the PDSR program by the British Council in Saudi Arabia.

\section{Competing interests}

The author declares that they have no competing interests.

Received: 12 February 2012 Accepted: 17 May 2012 Published: 17 May 2012

\section{References}

1. Kilbas, AA, Srivastava, HM, Trujillo, Jj: Theory and Applications of Fractional Differential Equations. Mathematics Studies. Elsevier, msterdam204 (2006)

2. Lakshmikantham, V, Leela, S, Devi, JV: Theory of Fractional Dynamic Systems. Cambridge Scientific Publishers, Cambridge (2009)

3. Diethelm, K: The Analysis of Fractional Differential Equations. Springer, Heidelberg (2010)

4. Agarwal, RP, Zhou, Y, He, Y: Existence of fractional neutral functional differential equations. Comput Math Appl. 59, 1095-1100 (2010). doi:10.1016/j.camwa.2009.05.010

5. Avad, HK, Glushak, AV: On perturbations of abstract fractional differential equations by nonlinear operators. J Math Sci. 170(3):306-323 (2010). doi:10.1007/s10958-010-0087-7

6. Kirane, M, Malik, SA: The profile of blowing-up solutions to a nonlinear system of fractional differential equations. Nonlinear Anal. 73, 3723-3736 (2010). doi:10.1016/..na.2010.06.088

7. Chai, G: Existence results for boundary value problems of nonlinear fractional differential equations. Comput Math Appl. 62, 2374-2382 (2011). doi:10.1016/j.camwa.2011.07.025

8. Zhang, S, Su, X: The existence of a solution for a fractional differential equation with nonlinear boundary conditions considered using upper and lower solutions in reverse order. Comput Math Appl. 62, 1269-1274 (2011). doi:10.1016/j. camwa.2011.03.008

9. Babakhani, A, Baleanu, D: Employing of some basic theory for class of fractional differential equations. Adv Diff Equ. 2011, 1-13 (2011)

10. Furati, KM, Kassim, MD, Tatar, Ne: Existence and uniqueness for a problem involving Hilfer fractional derivative. Comput Math Appl. (in press)

11. Podlubny, I: Fractional Differential Equations. Mathematics in Science and Engineering. Academic Press, San Diego198 (1999)

12. Hilfer, R: Applications of Fractional Calculus in Physics. World Scientific, Singapore (2000)

13. Caponetto, R, Dongola, G, Fortuna, L, Petráš, I: Fractional Order Systems: Modeling and Control Applications. World Scientific Series on Nonlinear Science. World Scientific72 (2010)

14. Monje, CA, Chen, Y, Vinagre, BM, Xue, D, Feliu, V: Fractional-order Systems and Controls. Adv Industr Control. Springer, New York (2010)

15. Hilfer, R: Experimental evidence for fractional time evolution in glass forming materials. Chem Phys. 284, 399-408 (2002). doi:10.1016/S0301-0104(02)00670-5

16. Wenchang, T, Wenxiao, P, Mingyu, X: A note on unsteady flows of a viscoelastic fluid with the fractional Maxwell model between two parallel plates. Int J Non-Linear Mech. 38, 645-650 (2003). doi:10.1016/S0020-7462(01)00121-4

17. Mainardi, F, Gorenflo, R: Time-fractional derivatives in relaxation processes: a tutorial survey. Fract Calc Appl Anal. 10(3):269-308 (2007)

18. Mainardi, F: Fractional Calculus and Waves in Linear Viscoelasticity. Imperial College Press, London (2010)

19. Baleanu, D, Mustafa, OG, Agarwal, RP: Asymptotically linear solutions for some linear fractional differential equations. Abstr Appl Anal. 2010, 8 (2010)

20. Sandev, T, Tomovski, Z: General time fractional wave equation for a vibrating string. J Phys A Math Theor. 43, 055204 (2010). doi:10.1088/1751-8113/43/5/055204

21. Klages, R, Radons, G, Sokolov, I: Anomalous Transport: Foundations and Applications. Wiley-VCH, Weinheim (2008)

22. Gerolymatou, E, Vardoulakis, I, Hilfer, R: Modelling infiltration by means of a nonlinear fractional diffusion model. J Phys D Appl Phys. 39, 4104-4110 (2006). doi:10.1088/0022-3727/39/18/022

23. Rao, BLSP: Statistical Inference for Fractional Diffusion Processes. Wiley, Chichester (2010)

24. Meerschaertm, MM, Sikorskii, A: Stochastic Models for Fractional Calculus. De Gruyter Studies in Mathematics. De Gruyter, Berlin43 (2012)

25. Hilfer, R, Anton, L: Fractional master equations and fractal time random walks. Phys Rev E. 51, R848-R851 (1995). doi:10.1103/PhysRevE.51.R848

26. Zhang, Y, Benson, DA, Meerschaert, MM, LaBolle, EM, Scheffler, HP: Random walk approximation of fractional-order multiscaling anomalous diffusion. Phys Rev E. 74, 026706-026715 (2006)

27. Baeumer, B, Meerschaert, MM, Nane, E: Brownian subordinators and fractional cauchy problems. Trans Am Math Soc. 361, 3915-3930 (2009). doi:10.1090/50002-9947-09-04678-9

28. Scalas, E, Gorenflo, R, Mainardi, F: Fractional calculus and continuous-time finance. Phys A. 284, 376-384 (2000). doi:10.1016/S0378-4371(00)00255-7

29. Scalas, E, Gorenflo, R, Mainardi, F, Meerschaert, M: Speculative option valuation and the fractional diffusion equation. Proceedings of the IFAC Workshop on Fractional Differentiation and its Applications, (FDA 04), Bordeaux (2004) 
30. Fulger, D, Scalas, E, Germano, G: Monte Carlo simulation of uncoupled continuous-time random walks yielding a stochastic solution of the space-time fractional diffusion equation. In Phys Rev E Stat, vol. 77, pp. 021122.Nonlinear Soft Matter Phys (2008)

31. Ortigueira, MD: Fractional Calculus for Scientists and Engineers. Lecture Notes in Electrical Engineering. Springer, Netherlands84 (2011)

32. Petráš, I: Fractional-Order Nonlinear Systems: Modeling, Analysis and Simulation. Springer, New York (2011)

33. Glushak, AV: Cauchy-type problem for an abstract differential equation with fractional derivative. Math Notes 77(1):26-38 (2005). Translated from Matematicheskie Zametki 77(1) 28-41 (2005). doi:10.1007/s11006-005-0003-5

34. Glushak, AV: On the properties of a Cauchy-type problem for an abstract differential equation with fractional derivatives. Math Notes 82(5):596-607 (2007). Translated from Matematicheskie Zametki 82(5), 665-677 (2007). doi:10.1134/S000143460711003X

35. Glushak, AV: Correctness of Cauchy-type problems for abstract differential equations with fractional derivatives. Russ Math 53(9):1-19 (2009). Translated from Izvestiya Vysshikh Uchebnykh sZavedenii. Matematika 2009(9), 13-24 (2009). doi:10.3103/S1066369X09090011

36. Samko, SG, Kilbas, AA, Marichev, Ol: Fractional Integrals and Derivatives, Theory and Applications. Gordon and Breach, Amsterdam (1993). Engl. Trans. from the Russian (1987)

37. Kou, C, Liu, J, Ye, Y: Existence and uniqueness of solutions for the Cauchy-type problems of fractional differential equations. Discr Dyn Nature Soc. 2010, 1-15 (2010)

38. Al-Jaser, A, Furati, KM: Singular fractional integro-differential inequalities and applications. J Inequal Appl. 2011, 110 (2011)

39. Kilbas, AA, Bonilla, B, Trujillo, JJ: Fractional integrals and derivatives, and differential equations of fractional order in weighted spaces of continuous functions (russian). Dokl Nats Akad Nauk Belarusi. 44(6):18-22 (2000)

doi:10.1186/1687-2770-2012-58

Cite this article as: Furati: A Cauchy-type problem with a sequential fractional derivative in the space of continuous functions. Boundary Value Problems 2012 2012:58.

\section{Submit your manuscript to a SpringerOpen ${ }^{\circ}$} journal and benefit from:

- Convenient online submission

- Rigorous peer review

- Immediate publication on acceptance

- Open access: articles freely available online

- High visibility within the field

- Retaining the copyright to your article

Submit your next manuscript at $\boldsymbol{\wedge}$ springeropen.com 